\title{
Nanowires and Nanorings at the Atomic Level
}

\author{
Midori Kawamura,* Neelima Paul, Vasily Cherepanov, and Bert Voigtländer ${ }^{\dagger}$ \\ Institut für Schichten und Grenzflächen ISG 3, Forschungszentrum Jülich, 52425 Jülich, Germany
}

(Received 27 March 2003; published 28 August 2003)

\begin{abstract}
The step-flow growth mode is used to fabricate $\mathrm{Si}$ and Ge nanowires with a width of $3.5 \mathrm{~nm}$ and a thickness of one atomic layer $(0.3 \mathrm{~nm})$ by self-assembly. Alternating deposition of Ge and Si results in the formation of a nanowire superlattice covering the whole surface. One atomic layer of Bi terminating the surface is used to distinguish between the elements $\mathrm{Si}$ and Ge. A difference in apparent height is measured in scanning tunneling microscopy images for $\mathrm{Si}$ and $\mathrm{Ge}$. Also, different kinds of twodimensional $\mathrm{Si} / \mathrm{Ge}$ nanostructures like alternating $\mathrm{Si}$ and $\mathrm{Ge}$ nanorings having a width of 5-10 $\mathrm{nm}$ were grown.
\end{abstract}

DOI: 10.1103/PhysRevLett.91.096102

Nanometer sized electronic structures are highly desirable for the future miniaturization of electronic devices. Nevertheless, nanostructuring is still a challenge and different approaches are followed [1,2]. Recently, nanowires have attracted a lot of interest because they are required to interconnect functional units in nano- and molecular electronics [3,4]. Nanowires smaller than the ones fabricated thus far are desirable. Because most electronic devices are fabricated on Si substrates, nanostructures grown epitaxially on $\mathrm{Si}$ substrates are most desirable.

One approach to growing two-dimensional nanowires would be to use preexisting steps as templates and deposit $\mathrm{Ge}$ and $\mathrm{Si}$ alternatingly in the step-flow growth mode. Since the deposited adatoms diffuse to, and are incorporated at the step edges this would result in twodimensional wires growing along the step edge. For the case of the important $\mathrm{Si} / \mathrm{Ge}$ system the observation of such a growth behavior may not be observed. This is the case because the initial deposition of $\mathrm{Ge}$ on $\mathrm{Si}$ is displacive, as experimentally found on the $\operatorname{Si}(001)$ [5,6]. Deposited $\mathrm{Ge}$ adatoms exchange sites with $\mathrm{Si}$ atoms at the surface, which leads to a homogenous mixed composition of $\mathrm{Si}$ and $\mathrm{Ge}$ atoms on the entire surface. This behavior prevents the formation of nanowires consisting of either Ge or Si during step-flow growth. Our approach is to prevent the displacive incorporation by the use of $\mathrm{Bi}$ terminating the surface.

Another key issue for the controlled fabrication of nanostructures consisting of different materials is a method of characterization which can distinguish between the different materials on the nanoscale. In the case of the system $\mathrm{Si} / \mathrm{Ge}$ it has been difficult to differentiate between $\mathrm{Si}$ and $\mathrm{Ge}$ due to their similar electronic structure. Only in rare cases it was possible to distinguish $\mathrm{Si}$ and $\mathrm{Ge}$ atoms $[7,8]$. In a recent approach to distinguish between $\mathrm{Si}$ and $\mathrm{Ge}$ atoms, a termination of the surface with $\mathrm{Cl}$ was used [8]. Since this termination was performed after growth it could not prevent the displacive adsorption of Ge. Therefore, Si and Ge atoms are located at random locations at the surface in this case.
PACS numbers: 68.65.-k, 68.55.Ac, 68.37.Ef

In this Letter we show that it is possible to grow $\mathrm{Si}$ and Ge nanowires with a width of $3.5 \mathrm{~nm}$ and a thickness of only one atomic layer $(0.3 \mathrm{~nm})$ by self-assembly. The cross section of these nanowires contains only about $21 \mathrm{Ge}$ atoms. These (sub)nanometer sized wires are grown epitaxially on the $\mathrm{Si}(111)$ substrate and are aligned along preexisting steps of monatomic height. Alternating deposition of $\mathrm{Ge}$ and $\mathrm{Si}$ can be used to grow a nanowire superlattice covering the whole surface. One atomic layer of Bi terminating the surface is used to prevent displacive adsorption and to distinguish between the elements $\mathrm{Si}$ and Ge. A difference in apparent height is measured in scanning tunneling microscopy (STM) images for $\mathrm{Si}$ and $\mathrm{Ge}$, respectively. Also, different kinds of twodimensional $\mathrm{Si} / \mathrm{Ge}$ nanostructures like alternating $\mathrm{Si}$ and Ge nanorings having a width of 5-10 $\mathrm{nm}$ were grown using self-assembly.

$\mathrm{Bi}, \mathrm{Ge}$, and $\mathrm{Si}$ were deposited on a clean Si(111) substrate by solid source molecular beam epitaxy. The deposition rate for $\mathrm{Bi}$ is $\sim 1 \mathrm{ML} / \mathrm{min}$ (1 ML corresponds to $1.56 \times 10^{15}$ atoms $/ \mathrm{cm}^{2}$ ). For $\mathrm{Si}$ and $\mathrm{Ge}$ deposition rates of $0.010 \mathrm{ML} / \mathrm{min}$ and $0.015 \mathrm{ML} / \mathrm{min}$ are used, respectively. The STM analysis was performed with a homebuilt beetle-type scanning tunneling microscope [6]. The sample bias voltages used are between +2.2 and $+2.6 \mathrm{~V}$ and the tunneling current $\sim 0.1 \mathrm{nA}$.

For the synthesis of nanowires we use preexisting step edges on the $\mathrm{Si}(111)$ surface as templates for the growth of Ge wires at the step edges. When the diffusion of the deposited atoms is sufficient to reach the step edges, these deposited atoms are incorporated exclusively at the step edges and the growth proceeds by a homogenous advancement of the steps (step-flow growth mode) [9]. If small amounts of $\mathrm{Ge}$ are deposited, the steps advance only some nanometers and narrow Ge wires can be grown. The surface is terminated with a monolayer of Bi before the growth of $\mathrm{Si}$ or $\mathrm{Ge}$. As will be shown in the following, this Bi termination prevents the displacive incorporation of $\mathrm{Ge}$ and opens the possibility to distinguish between $\mathrm{Si}$ and Ge. Figure 1(a) shows an STM image after repeated alternating deposition of 0.15 atomic layers of $\mathrm{Ge}$ and $\mathrm{Si}$, 

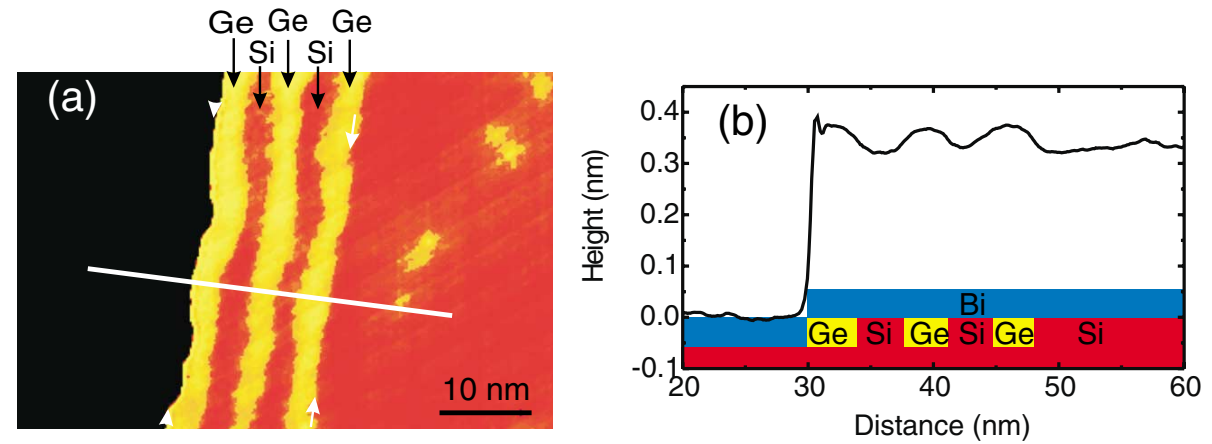

(c)

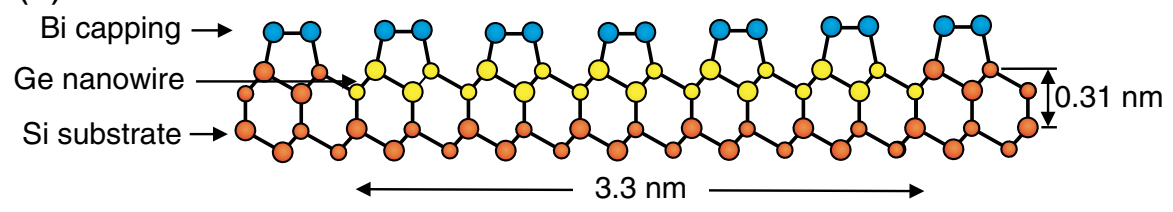

FIG. 1 (color online). (a) STM image of two-dimensional Ge/Si nanowires grown by step flow at a preexisting step edge on a Bi terminated $\mathrm{Si}(111)$ substrate. Si wires (dark) and Ge wires (light) can be distinguished by different apparent heights. (b) The cross section along the white line shows the dimensions of the Si and Ge nanowires. The width of the wires is $\sim 3.5 \mathrm{~nm}$ and the height is only one atomic layer $(0.3 \mathrm{~nm})$. (c) Atomic structure of a $3.3 \mathrm{~nm}$ wide Ge wire (light circles) on the Si substrate (dark circles) capped by $\mathrm{Bi}$ (circles in the top layer). The cross section of the Ge wire contains only $21 \mathrm{Ge}$ atoms.

respectively. One atomic layer of $\mathrm{Bi}$ was deposited at $740 \mathrm{~K}$ and alternating $\mathrm{Ge}$ and $\mathrm{Si}$ deposition of $0.15 \mathrm{ML}$ was performed at $T=720$ and $750 \mathrm{~K}$, respectively, under the continuous flux of $\mathrm{Bi}$.

Because of the step-flow growth, Ge and Si wires are formed at the advancing step edge. Both elements can be easily distinguished by the apparent heights in the STM image [Fig. 1(a)]. It turned out that the height measured by the STM is higher on areas consisting of Ge (light stripes) than on areas consisting of Si (dark stripes). The assignment of $\mathrm{Ge}$ and $\mathrm{Si}$ wires is evident from the order of the deposited materials $(\mathrm{Ge}, \mathrm{Si}, \mathrm{Ge}, \mathrm{Si}$, and $\mathrm{Ge}$, respectively, in this case). The initial step position is indicated by white arrows in the right-hand part of Fig. 1(a). The step edge has advanced towards the left [arrowheads in Fig. 1(a)] after the growth of the nanowires.

The apparent height of $\mathrm{Ge}$ areas is $\sim 0.07 \mathrm{~nm}$ higher than the apparent height of Si wires [Fig. 1(b)]. The width of the $\mathrm{Si}$ and $\mathrm{Ge}$ wires is $\sim 3.5 \mathrm{~nm}$ as measured from the cross section [Fig. 1(b)]. The nanowires are two-dimensional with a height of only one atomic layer $(\sim 0.3 \mathrm{~nm})$. Therefore, the cross section of a $3.3 \mathrm{~nm}$ wide Ge nanowire contains only 21 atoms [Fig. 1(c)]. In spite of the subnanometer thickness the atoms building the Ge nanowire are connected by strong lateral covalent bonds [Fig. 1(c)]. In this respect these nanowires are different from single dimer rows of $\mathrm{Ge}$ or $\mathrm{Bi}$ on $\mathrm{Si}(001)$ which are sometimes considered as nanowires [10]. However, those wires have no lateral $\mathrm{Ge}-\mathrm{Ge}$ or $\mathrm{Bi}-\mathrm{Bi}$ bonds, but only bonds to the underlying substrate.

DC current heating during cleaning of the Si substrate was used to form an array of equidistant steps [11] [Fig. 2(a)]. The $\mathrm{Si} / \mathrm{Ge}$ wires are homogenous in width over larger distances and have a length of several thousand $\mathrm{nm}$. Different width of the wires can be easily achieved by different amounts of Ge and Si deposited. Figure 2(b) shows an array of nine nanowires each $\sim 3-5 \mathrm{~nm}$ wide grown at each step edge (Ge coverage: $0.10 \mathrm{ML}$, Si coverage: $0.15 \mathrm{ML}$ ). Here the total deposited amount was chosen in a way to cover the whole surface area by a superlattice of nanowires.

Bi floats always on top of the growing layer, which is a usual behavior in surfactant mediated epitaxy [12]. The
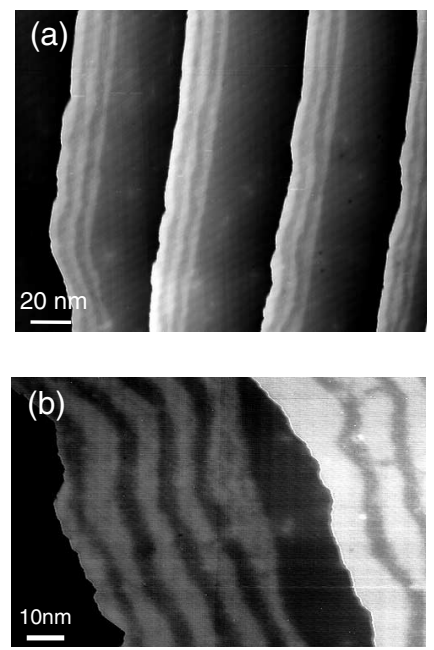

FIG. 2. (a) $\mathrm{Si} / \mathrm{Ge}$ nanowires on a larger scale growing at four step edges. The homogeneity of the nanowires is visible in this STM image. (b) Alternating deposition of nine wires per step edge results in the formation of a superlattice of $\mathrm{Si} / \mathrm{Ge}$ nanowires covering the whole surface. 
Bi termination of the surface also suppresses a displacive incorporation of $\mathrm{Ge}$ (and $\mathrm{Si}$ ) during the growth of the wires. For a purely displacive incorporation a homogenous $\mathrm{SiGe}$ surface alloy rather than $\mathrm{Si} / \mathrm{Ge}$ wires would form due to the large diffusion length in step-flow growth. Experimentally we do not find an indication even for a partially displacive incorporation of $\mathrm{Ge}$ and $\mathrm{Si}$. In such a case the Ge content in the Ge wires would increase with the Ge coverage and the $\mathrm{Si}$ content in the $\mathrm{Si}$ wires would decrease after deposition of several $\mathrm{Si} / \mathrm{Ge}$ wires, due to the increasing Ge coverage in the surface layer. An analysis of images like that in Fig. 2(b) shows no change in the height difference between neighboring wires with increasing $\mathrm{Ge}$ coverage.

In the following it will be shown that, using twodimensional island growth, different types of $\mathrm{Si} / \mathrm{Ge}$ nanostructures, namely $\mathrm{Si} / \mathrm{Ge}$ ring structures, can be grown by self-assembly [Fig. 3(a)]. In the twodimensional island growth mode the diffusion of deposited atoms at the surface is reduced so that most of the diffusing atoms do not reach the step edges but nucleate as two-dimensional islands or attach to existing islands. Once atomic layer high islands have nucleated, deposited atoms diffuse towards the island edge and are incorporated forming a new ring of $\mathrm{Ge}$ or $\mathrm{Si}$, respectively [Fig. 3(a)]. The measured height of the Ge rings is $0.09 \mathrm{~nm}$ higher than the measured height of the Si rings [Fig. 3(b)]. The width of the rings is $5-10 \mathrm{~nm}$ and the thickness is only one atomic layer $(0.3 \mathrm{~nm})$. The $\mathrm{Si} / \mathrm{Ge}$ ring structure shown in Fig. 3(a) was obtained as follows. The starting surface is the clean $\operatorname{Si}(111)-(7 \times 7)$ surface which is initially covered by a one atomic layer thick $\mathrm{Bi}$ layer at $740 \mathrm{~K}$. The change of the surface reconstruction from $(7 \times 7)$ to $(\sqrt{3} \times \sqrt{3})$ (due to the Bi coverage) releases about 0.1 atomic layers of surface $\mathrm{Si}$ atoms which form two-dimensional $\mathrm{Si}$ islands [13]. These $\mathrm{Si}$ islands form the cores (diameter $10-20 \mathrm{~nm}$ ) of the $\mathrm{Si} / \mathrm{Ge}$ ring structures. Subsequent alternating deposition of $\mathrm{Ge}$ and $\mathrm{Si}$ results in the formation of the $\mathrm{Si} / \mathrm{Ge}$ ring structures around the $\mathrm{Si}$ core $(\mathrm{Ge}$ and $\mathrm{Si}$ deposition of $0.15 \mathrm{ML}$ was performed at $T=720$ and $750 \mathrm{~K}$, respectively). The growth conditions for $\mathrm{Ge}$ and $\mathrm{Si}$ growth were chosen in a way that no additional $\mathrm{Si}$ or $\mathrm{Ge}$ islands nucleate, but all deposited atoms are incorporated in the ring structures.

There are several possible reasons for the observed height difference for $\mathrm{Si}$ and $\mathrm{Ge}$ areas. The measured height difference corresponds to a $\sim 25 \%$ higher height of the $\mathrm{Ge}$ atomic layer than the $\mathrm{Si}$ atomic layer. This height difference cannot be explained by vertical relaxation reliving the strain induced due to the $4.2 \%$ larger lattice constant of $\mathrm{Ge}$ compared to $\mathrm{Si}$ [14]. Another possible explanation for the different heights would be a different surface reconstruction of the terminating $\mathrm{Bi}$ layer on $\mathrm{Si}$ and $\mathrm{Ge}$. The atomic structure of the Bi termination layer on $\mathrm{Si}$ is known to consist of $\mathrm{Bi}$ trimers [15]. In atomic resolved images such as Fig. 3(c) the same $(\sqrt{3} \times \sqrt{3})$ reconstruction is observed both on the $\mathrm{Si}$ and
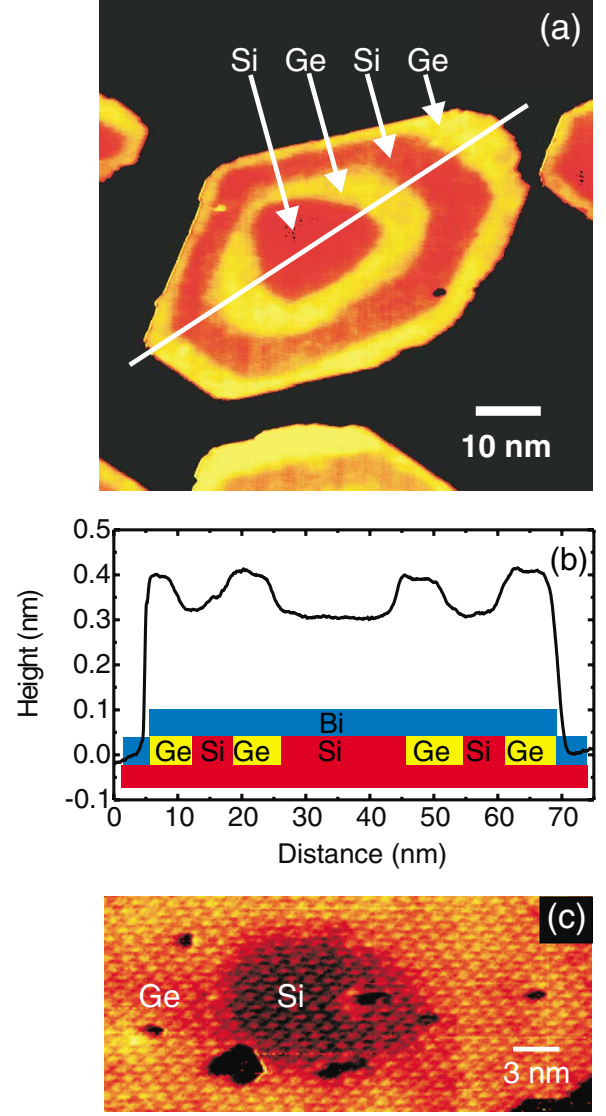

FIG. 3 (color online). (a) Two-dimensional Ge/Si ring structure imaged with the STM. Ge rings are shown as light and Si rings shown darker. The width of the rings is $5-10 \mathrm{~nm}$ and the height is one atomic layer $(0.3 \mathrm{~nm})$. (b) Cross section along the line indicated in (a). Because of the Bi termination the $\mathrm{Ge}$ rings are imaged $\sim 0.09 \mathrm{~nm}$ higher than the Si rings. A schematic of the ring structure is shown in the inset. (c) An atomic resolved image shows the same $(\sqrt{3} \times \sqrt{3})$ structure at the Si core and the Ge ring. The protrusions indicate the Bi trimers.

the Ge areas. Therefore, it seems improbable that a different surface reconstruction on $\mathrm{Si}$ and $\mathrm{Ge}$ is the reason for the different heights in the STM images. The remaining explanation is a different electron density of states for $\mathrm{Bi}$ bond to $\mathrm{Si}$ and $\mathrm{Ge}$, respectively. This can lead to the observed different apparent heights. This explanation is confirmed by the fact that the measured height difference shows a pronounced dependence on the applied bias voltage.

The atomic resolved STM image of two Ge wires and one Si wire in Fig. 4(a) shows more details on the growth of $\mathrm{Ge}$ and $\mathrm{Si}$ nanowires. The brighter protrusions visible in this image are due to Bi trimers arranged in a $(\sqrt{3} \times$ $\sqrt{3}$ ) superstructure [15] on top of the Ge or Si nanowire, schematically shown in Fig. 1(c) [only two of the three atoms in each trimer are visible due to the projection along the $(11 \overline{0})$ direction]. While the border from Si to Ge is almost atomically sharp [arrows in Fig. 4(a)], the border from $\mathrm{Ge}$ to $\mathrm{Si}$ is more fuzzy [arrowheads in 

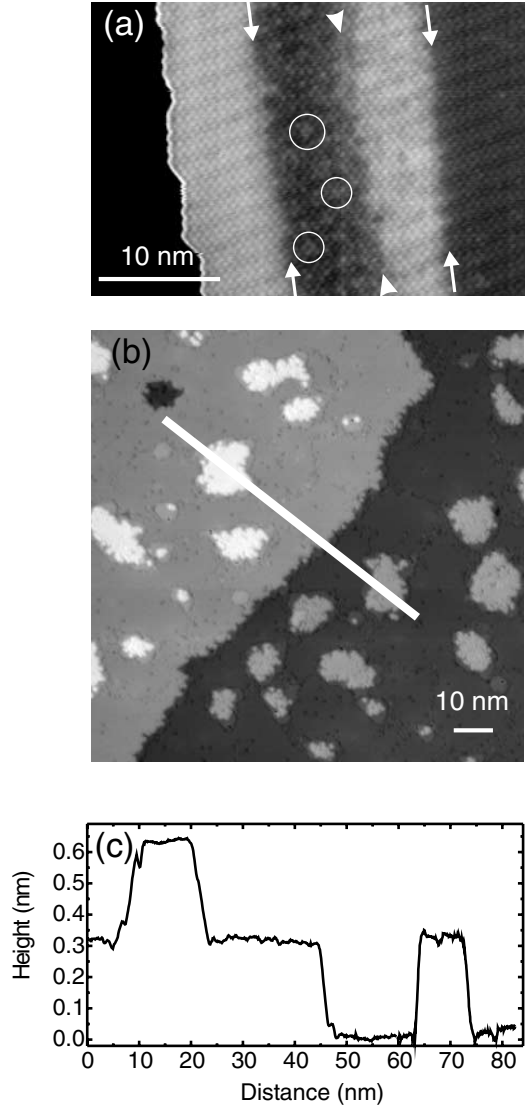

FIG. 4. (a) Atomically resolved image of $\mathrm{Ge} / \mathrm{Si}$ nanowires on Bi terminated $\mathrm{Si}(111)$ surfaces. The initial $\mathrm{Si} / \mathrm{Ge}$ boundary is nearly atomically sharp (right arrows), while at the $\mathrm{Ge} / \mathrm{Si}$ boundary (arrow heads) a small amount of interdiffusion is visible. (b) Submonolayer Ge deposition on a Sb terminated Si(111) surface. (c) No other height than the Si step height is observed at the Ge islands, or at the step edge. This shows, that the distinction between $\mathrm{Si}$ and $\mathrm{Ge}$ by the apparent height in the STM image is specific to Bi terminating the surface.

Fig. 4(a)]. Some brighter trimers are found in the Si wire between the two Ge wires [circles in Fig. 4(a)]. This can be explained by diffusion of $\mathrm{Ge}$ atoms into the $\mathrm{Si}$ wire. However, since the brighter trimers in the silicon wires are still much darker than the trimers in the Ge wires, only some of the six $\mathrm{Si}$ atoms below a $(\sqrt{3} \times \sqrt{3})$ unit cell are replaced by $\mathrm{Ge}$ atoms. To prevent such a partial diffusion of $\mathrm{Ge}$ to the $\mathrm{Si}$ wires, the nanowires could be grown at lower temperatures.

Control experiments were performed to determine if the observed height difference between two-dimensional $\mathrm{Si}$ and $\mathrm{Ge}$ nanostructures is specific to the Bi termination of the surface. After growth of Ge on $\mathrm{Si}(111)$ without any other element terminating the surface, we found no height differences other than the Si step height, in accord with various other studies. After terminating the surface with $\mathrm{Sb}$, which also results in the formation of a $(\sqrt{3} \times \sqrt{3})$ trimer structure, and subsequent growth of $0.2 \mathrm{ML} \mathrm{Ge}$ at $770 \mathrm{~K}$ and a growth rate of $0.5 \mathrm{ML} / \mathrm{min}$ [Fig. 4(b)], no height differences other than the Si step height could be found at the step edges or on islands [Fig. 4(c)]. These are deposition conditions under which a height difference was found on the Bi terminated surface. This shows that only the specific electronic structure of $\mathrm{Bi}$ gives rise to the different apparent heights measured in the STM images.

In summary, we have shown that the controlled formation of different kinds of two-dimensional $\mathrm{Si} / \mathrm{Ge}$ nanostructures, like nanowires, nanowire superlattices, and nanorings, is feasible. The nanostructures grown have a width down to $3.5 \mathrm{~nm}$ and a subnanometer thickness $(0.3 \mathrm{~nm})$, corresponding to a cross section consisting of only $\sim 21$ atoms. In spite of the subnanometer thickness the atoms building the Ge nanostructures are connected by strong lateral covalent bonds. A combination of nanorings and nanowires could be used to build even more complex structures. The controlled formation of such small nanostructures would be impossible without a material specific characterization method on the nanoscale. A simple and general method has been presented to distinguish between $\mathrm{Si}$ and Ge in two-dimensional nanostructures using the height difference in STM images after termination of the surface with Bi.

*Permanent address: Department of Materials Science, Kitami Institute of Technology, 165 Koen-cho, Kitami, Hokkaido 090-8507, Japan.

${ }^{\dagger}$ Corresponding author.

Electronic address: b.voigtlaender@fz-juelich.de

[1] K. Alchalabi, D. Zimin, G. Kostorz, and H. Zogg, Phys. Rev. Lett. 90, 026104 (2003).

[2] E. S. Kim, N. Usami, and Y. Shiraki, Appl. Phys. Lett. 72, 1617 (1998).

[3] L. J. Lauhon, M. S. Gudiksen, D. Wang, and C. M. Lieber, Nature (London) 420, 57 (2002).

[4] P. Kluth, Q. T. Zhao, S. Winnerl, S. Lenk, and S. Mantl, Appl. Phys. Lett. 79, 824 (2001).

[5] R. M. Tromp, Phys. Rev. B 47, 7125 (1993).

[6] B. Voigtländer, Surf. Sci. Rep. 43, 127 (2001).

[7] X. R. Qin, B. S. Swartzentruber, and M. G. Lagally, Phys. Rev. Lett. 84, 4645 (2000).

[8] D.-S. Lin, J.-L. Wu, S.-Y. Pan, and T. C. Chiang, Phys. Rev. Lett. 90, 046102 (2003).

[9] B. Voigtländer, Th. Weber, P. Smilauer, and D. E. Wolf, Phys. Rev. Lett. 78, 2164 (1997).

[10] J. H. G. Owen, K. Miki, H. Koh, H.W. Yeom, and D. R. Bowler, Phys. Rev. Lett. 88, 226104 (2002).

[11] Y.- N. Yang, E. S. Fu, and E. S. Williams, Surf. Sci. 356, 101 (1996).

[12] M. Copel, M. C. Reuter, E. Kaxiras, and R. M. Tromp, Phys. Rev. Lett. 63, 632 (1989).

[13] R. G. Ryland, S. Hasegawa, and E. D. Williams, Surf. Sci. 345, 222 (1996).

[14] A biaxial compression of $4.2 \%$ can lead to a maximum expansion in the growth direction of $8.4 \%$ for a Poisson ratio of 0.5 .

[15] S. Kono, Surf. Rev. Lett. 1, 359 (1994). 